\title{
REMARKS ON THE INITIAL VALUE PROBLEM OF THE GENERAL PARTIAL DIFFERENTIAL EQUATION OF THE FIRST ORDER
}

BY H. R. COOLEY

1. Introduction. This paper presents a new approach to the classical theory of partial differential equations of the first order by establishing the equivalence of the initial value problem to another problem associated with a system of quasi-linear partial differential equations of the first order. All the equations of this quasi-linear system will have the same "principal part"; this means that the derivatives have the same coefficients in all the equations. Incidentally, systems of differential equations with the same principal part are assuming an important rôle in the theory of partial differential equations.*

In the case of quasi-linear systems the theory of integration can, as we shall see, be reduced to that of the integration of a system of ordinary differential equations in the same way as with a single linear partial differential equation. We shall then show that the initial value problem of the general partial differential equation of the first order is equivalent to a certain initial value problem of our quasi-linear system with identical principal parts. In this way we can develop the theory of characteristics and the solution of the initial value problem for a general partial differential equation of the first order.

2. Systems of Quasi-Linear Partial Differential Equations of the First Order with Identical Principal Parts. Let

$$
\sum_{i=1}^{k} a_{i} \frac{\partial u_{\mu}}{\partial x_{i}}=b_{\mu}, \quad(\mu=1, \cdots, m),
$$

be a system of quasi-linear partial differential equations in which the coefficients $a_{i}$ are the same in all equations. This is a system of quasi-linear equations with identical principal parts for $m$ functions $u_{1}, \cdots, u_{m}$ of $k$ variables $x_{1}, \cdots, x_{k}$. In these equations $a_{i}$ and $b_{\mu}$ are functions of $x_{1}, \cdots, x_{k}, u_{1}, \cdots, u_{m}$.

* For this topic see Courant-Hilbert, Methoden der Mathematischen Physik, vol. II, Chapter 2, appendix and Chapter 5 (in press). 
If now, generalizing a well-known procedure, ${ }^{*}$ instead of requiring $m$ functions $u_{\mu}$ explicitly, we require $m$ implicit relations,

$$
\phi_{\mu}\left(x_{1}, \cdots, x_{k}, u_{1}, \cdots, u_{m}\right)=c_{\mu}, \quad(\mu=1, \cdots, m),
$$

depending on $m$ parameters $c_{\mu}$, the system (1) is transformed into a system of homogeneous linear partial differential equations with identical principal parts, or, what amounts to the same thing, a single homogeneous linear partial differential equation for which we require $m$ independent solutions. For these functions $\phi_{\mu}$, we have

$$
\frac{\partial \phi_{\mu}}{\partial x_{i}}+\sum_{\lambda=1}^{m} \frac{\partial \phi_{\mu}}{\partial u_{\lambda}} \frac{\partial u_{\lambda}}{\partial x_{i}}=0 .
$$

Multiplying by $a_{i}$ and adding, we obtain

$$
\sum_{i=1}^{k} a_{i} \frac{\partial \phi_{\mu}}{\partial x_{i}}+\sum_{i=1}^{k} \sum_{\lambda=1}^{m} \frac{\partial \phi_{\mu}}{\partial u_{\mu}} a_{i} \frac{\partial u_{\lambda}}{\partial x_{i}}=0 .
$$

Since $\sum_{i=1}^{k} a_{i} \partial u_{\lambda} / \partial x_{i}=b_{\lambda}$, we have

$$
\sum_{i=1}^{k} a_{i} \frac{\partial \phi_{\mu}}{\partial x_{i}}+\sum_{\lambda=1}^{m} b_{\lambda} \frac{\partial \phi_{\mu}}{\partial u_{\lambda}}=0 .
$$

If we let $b_{\lambda}=a_{k+\lambda}, u_{\lambda}=x_{k+\lambda}, \phi_{\mu}=\phi, k+m=r$, equation (3) becomes

$$
\sum_{\rho=1}^{r} a_{\rho} \frac{\partial \phi}{\partial x_{\rho}}=0 .
$$

Equation (4) is a homogeneous linear partial differential equation and is equivalent to the system of ordinary differential equations

$$
\frac{d x_{\rho}}{d s}=a_{\rho}, \quad \frac{d \phi}{d s}=0,
$$

which are its characteristic equations. By integrating this system of characteristic equations a solution of (4), depending on $r$ independent parameters, can be found.

In order to show the equivalence of system (1) and equation (4), in addition to the fact that a solution of (1) satisfies (3) in

* Cf. Courant-Hilbert, loc. cit., Chapter 1. 
accord with the above derivation, it is necessary to show that, conversely, $m$ independent solutions of (4) furnish a solution of (1).

Let $\phi_{1}, \cdots, \phi_{m}$ be $m$ independent solutions of (4). Then $\Delta=\left|\partial \phi_{\mu} / \partial u_{\lambda}\right| \neq 0$, and hence from the equations $\phi_{\mu}=c_{\mu}$ we can obtain

$$
u_{\mu}=u_{\mu}\left(x_{1}, \cdots, x_{k}, c_{1}, \cdots, c_{m}\right) .
$$

These functions $u_{\mu}$ must satisfy (1). For, differentiating

$$
\phi_{\mu}\left(x_{1}, \cdots, x_{k}, u_{1}, \cdots, u_{m}\right)=c_{\mu},
$$

we obtain (2). Using this equation with (3), we have

or

$$
\sum_{\lambda=1}^{m} b_{\lambda} \frac{\partial \phi_{\mu}}{\partial u_{\lambda}}=-\sum_{i=1}^{k} a_{i} \frac{\partial \phi_{\mu}}{\partial x_{i}}=\sum_{i=1}^{k} \sum_{\lambda=1}^{m} \frac{\partial \phi_{\mu}}{\partial u_{\lambda}} a_{i} \frac{\partial u_{\lambda}}{\partial x_{i}},
$$

$$
\sum_{\lambda=1}^{m} b_{\lambda} \frac{\partial \phi_{\mu}}{\partial u_{\lambda}}-\sum_{\lambda=1}^{m} \frac{\partial \phi_{\mu}}{\partial u_{\lambda}} \sum_{i=1}^{k} a_{i} \frac{\partial u_{\lambda}}{\partial x_{i}}=0 .
$$

Hence

$$
\sum_{\lambda=1}^{m} \frac{\partial \phi_{\mu}}{\partial u_{\lambda}}\left\{b_{\lambda}-\sum_{i=1}^{k} a_{i} \frac{\partial u_{\lambda}}{\partial x_{i}}\right\}=0 .
$$

Since $\Delta \neq 0$, it follows that $b_{\lambda}-\sum_{i=1}^{k} a_{i} \partial u_{\lambda} / \partial x_{i}=0$, that is, (1) is satisfied.

3. The Derivation of a System of the Form of (1) from the General Partial Differential Equation of the First Order. If the number of independent variables is $k$, the general partial differential equation of the first order is

$$
F\left(x_{1}, \cdots, x_{k}, u, p_{1}, \cdots, p_{k}\right)=0 ; \quad p_{i}=\frac{\partial u}{\partial x_{i}} .
$$

Differentiating (5) with respect to $x_{\lambda}$, and making use of the relations $\partial p_{i} / \partial x_{j}=\partial p_{j} / \partial x_{i}$, we obtain the first $k$ equations of the system

$$
\left\{\begin{array}{l}
\text { (a) } \sum_{i=1}^{k} \frac{\partial F}{\partial p_{i}} \frac{\partial p_{\lambda}}{\partial x_{i}}=-\frac{\partial F}{\partial x_{\lambda}}-\frac{\partial F}{\partial u} p_{\lambda}, \quad(\lambda=1, \cdots, k), \\
\text { (b) } \sum_{i=1}^{k} \frac{\partial F}{\partial p_{i}} \frac{\partial u}{\partial x_{i}}=\sum_{i=1}^{k} \frac{\partial F}{\partial p_{i}} p_{i} .
\end{array}\right.
$$


The last equation is a formal triviality depending on the relation $\partial u / \partial x_{i}=p_{i}$, written down to complete the system. This system is of the form (1), in which

$$
\left.\begin{array}{rlrl}
x_{i} & =x_{i}, & u_{\mu} & =p_{\mu}, \\
a_{i} & =\frac{\partial F}{\partial p_{i}}, & b_{\mu} & =-\frac{\partial F}{\partial x_{\mu}}-\frac{\partial F}{\partial u} p_{\mu},
\end{array}\right\}, \quad(\mu=1, \cdots, k) .
$$

The characteristic equations in this case are

$$
\begin{array}{r}
\frac{d x_{i}}{d s}=\frac{\partial F}{\partial p_{i}}, \quad \frac{d u}{d s}=\sum_{i=1}^{k} \frac{\partial F}{\partial p_{i}} p_{i}, \quad \frac{\partial u_{\mu}}{d s}=-\frac{\partial F}{\partial x_{\mu}}-\frac{\partial F}{\partial u} p_{\mu}, \\
(i, \mu=1, \cdots, k) .
\end{array}
$$

4. Solution of the Initial Value Problem of the General Partial Differential Equation of the First Order. The initial value problem for equation (5) is the following: We are given an initial manifold, $M\left(x_{1}, x_{2}, \cdots, x_{k}\right)=0$, with a set of functions $u$, $p_{1}, \cdots, p_{k}$, satisfying the relations

$$
d u=\sum_{i=1}^{k} p_{i} d x_{i},
$$

which, for $\partial M / \partial x_{k} \neq 0$, is equivalent to the $k-1$ relations

and

$$
\left(\frac{\partial u}{\partial x_{\nu}}-p_{\nu}\right) \frac{\partial M}{\partial x_{k}}-\left(\frac{\partial u}{\partial x_{k}}-p_{k}\right) \frac{\partial M}{\partial x_{\nu}}=0, \quad(\nu<k) ;
$$

$$
F\left(x_{1}, \cdots, x_{k}, u, p_{1}, \cdots, p_{k}\right)=0
$$

on $M=0$. These initial data are assumed to be non-characteristic; this means that for the initial values

$$
\sum_{i=1}^{k} \frac{\partial F}{\partial p_{i}} \frac{\partial M}{\partial x_{i}} \neq 0
$$

holds. Then we seek a function $u\left(x_{1}, \cdots, x_{k}\right)$ satisfying the differential equation (5), which has the values prescribed for $u$ on $M$, and for which the derivatives $\partial u / \partial x_{i}$ have the prescribed initial values $p_{i}$ on $M$. 
Again in the initial value problem for the system (6) the values of $u$ and $p_{i}$ are prescribed on the initial manifold $M$, subject to the same condition (9) in order that $M$ be non-characteristic.

Now our statement is as follows: If in this second problem the additional conditions (7) and (8) are satisfied, then this problem becomes equivalent to the first problem for equation (5), and by solving this second problem for the quasi-linear system we have solved the problem for the general equation.

The equivalence of the initial value problem of the general equation (5) and the special initial value problem of the system (6) can be shown in the following manner.

First we note that a solution of the initial value problem of (5) yields a solution $u, p_{i}=\partial u / \partial x_{i}$, of the corresponding initial value problem of the system (6), since if $u=u\left(x_{1}, \cdots, x_{k}\right)$ satisfies (5) identically, then (6) follows immediately by differentiation.

Now let $u\left(x_{1}, \cdots, x_{k}\right), p_{i}\left(x_{1}, \cdots, x_{k}\right)$, in which $i=1, \cdots, k$, be a solution of the restricted initial value problem of (6). We first observe that from (6b) and (7) we can infer that on the initial manifold $M$ the relations $\partial u / \partial x_{i}=p_{i}$ hold, since the determinant of the linear equations $(6 \mathrm{~b})$ and $(7)$ for $\partial u / \partial x_{i}-p_{i}$ is (except for a factor) the left side of (9), and thus does not vanish. Furthermore from (8) we have $F=0$ on $M$. We now have to show that $F=0, \partial u / \partial x_{i}=p_{i}$, everywhere.

Let

$$
\begin{gathered}
F\left(x_{1}, \cdots, x_{k}, u, p_{1}, \cdots, p_{k}\right)=R\left(x_{1}, \cdots, x_{k}\right), \\
\frac{\partial u}{\partial x_{i}}-p_{i}=P_{i}\left(x_{1}, \cdots, x_{k}\right) .
\end{gathered}
$$

We have $R=P_{i}=0$ on the initial manifold. Differentiating $R$ with respect to $x_{i}$, and rewriting equations (6), we have

$$
\left\{\begin{aligned}
\frac{\partial R}{\partial x_{i}} & =\frac{\partial F}{\partial x_{i}}+F_{u} \frac{\partial u}{\partial x_{i}}+\sum_{j=1}^{k} \frac{\partial F}{\partial p_{j}} \frac{\partial p_{j}}{\partial x_{i}}, \\
0 & =\frac{\partial F}{\partial x_{i}}+F_{u} p_{i}+\sum_{j=1}^{k} \frac{\partial F}{\partial p_{i}} \frac{\partial p_{i}}{\partial x_{j}}, \quad(i=1, \cdots, k) . \\
0 & =\sum_{j=1}^{k} \frac{\partial F}{\partial p_{j}} p_{j}-\sum_{j=1}^{k} \frac{\partial F}{\partial p_{i}} \frac{\partial u}{\partial x_{j}},
\end{aligned}\right.
$$


Multiplying these $2 k+1$ equations respectively by

$$
\frac{\partial F}{\partial p_{1}}, \ldots, \frac{\partial F}{\partial p_{k}},-\frac{\partial F}{\partial p_{1}}, \ldots,-\frac{\partial F}{\partial p_{k}}, F_{u},
$$

and adding, we obtain

$$
\sum_{i=1}^{k} \frac{\partial F}{\partial p_{i}} \frac{\partial R}{\partial x_{i}}=0
$$

This is a homogeneous linear partial differential equation for $R$, with $R=0$ on the initial manifold. Therefore $R \equiv 0$.

The last of equations (10) can be written

$$
\sum_{j=1}^{k} \frac{\partial F}{\partial p_{j}} P_{j}=0
$$

Making use of the fact that $\partial R / \partial x_{i}=0$, and subtracting the $(k+i)$ th equation from the $i$ th equation, in system (10), we obtain the system

$$
F_{u}\left(\frac{\partial u}{\partial x_{i}}-p_{i}\right)+\sum_{j=1}^{k} \frac{\partial F}{\partial p_{j}}\left(\frac{\partial p_{j}}{\partial x_{i}}-\frac{\partial p_{i}}{\partial x_{j}}\right)=0,(i=1, \cdots, k),
$$

or

$$
F_{u} P_{i}+\sum_{j=1}^{k} \frac{\partial F}{\partial p_{j}}\left(\frac{\partial P_{i}}{\partial x_{j}}-\frac{\partial P_{j}}{\partial x_{i}}\right)=0 .
$$

If we differentiate (11) with respect to each of the independent variables and substitute in (12), system (12) then becomes

$$
\sum_{j=1}^{k} \frac{\partial F}{\partial p_{j}} \frac{\partial P_{i}}{\partial x_{j}}=A_{i}
$$

in which $A_{i}$ is a homogeneous linear form in $P_{1}, \cdots, P_{k}$. The left-hand side of each equation of (13) is the directional derivative of $P_{i}, \partial P_{i} / \partial \sigma$, in the direction determined by

$$
\frac{\partial x_{j}}{\partial \sigma}=\frac{\partial F}{\partial p_{j}},
$$

where $\sigma$ is a parameter of the characteristic curve through the point under consideration. Hence (13) may be written 


$$
\frac{\partial P_{i}}{\partial \sigma}=A_{i} .
$$

This system, together with the initial conditions, is satisfied by $P_{i}=0,(i=1, \cdots, k)$. Hence, on account of the uniqueness of the solution of (14) with given initial values, we conclude that $P_{i} \equiv 0$, and the proof is complete.

New York UnIVersity

\title{
ON THE EXISTENCE OF LINEAR FUNCTIONALS D EFINED OVER LINEAR SPACES*
}

\author{
BY R. P. AGNEW
}

1. Introduction. A function $q(x)$ with domain in a linear space $E$ and range in the set $R$ of real numbers is called a functional, and $q(x)$ is called linear, if

$$
q(a x+b y)=a q(x)+b q(y), \quad x, y \varepsilon E ; a, b \varepsilon R .
$$

We call a functional $r(x)$ an $r$-function (over $E$ ) if there exists a linear functional $f(x)$ with

$$
f(x) \leqq r(x), \quad \quad x \varepsilon E .
$$

Using a notation of Banach $\uparrow$ we call a functional $p(x)$ a $p$-function if

$$
\begin{aligned}
p(t x) & =t p(x), & t \geqq 0, x_{\varepsilon} E, \\
p(x+y) & \leqq p(x)+p(y), & x, y \varepsilon E .
\end{aligned}
$$

A fundamental theorem of Banach (loc. cit., p. 29) can be stated as follows:

THEOREM (Banach). Each p-function is an r-function.

In some problems $\ddagger$ involving existence of linear functionals $f_{1}(x)$ having prescribed properties, there appears a functional $q(x)$ with the following significance: There exists a linear functional $f_{1}$ having the requisite properties if and only if there exists

* Presented to the Society, September 8, 1937.

$\dagger$ S. Banach, Théorie des Opérations Linéaires, Warsaw, 1932, p. 28.

$¥$ The author intends to discuss these problems at some future time. 